\title{
ENVOLVIMENTO HEPÁTICO NA SARCOIDOSE: RELATO DE CASO*
}

\author{
André Albernaz ${ }^{1}$, Ana Cláudia Ferreira Rosa ${ }^{2}$, Márcio Martins Machado ${ }^{3}$, Nestor de Barros ${ }^{4}$, \\ Orlando Milhomem da Mota ${ }^{5}$, Jales Benevides Santana Filho ${ }^{6}$, Rodrigo Alvarenga Nunes ${ }^{7}$, \\ Huber Martins Vasconcelos Júnior ${ }^{8}$, Patrícia Tavares Pereira de Souza ${ }^{8}$, Thaís de Toledo Lima ${ }^{8}$, \\ Leonardo Medeiros Milhomem ${ }^{9}$, Carlos Alberto Ximenes Filho ${ }^{2}$, Carlos Alberto Ximenes ${ }^{10}$, \\ Giovanni Guido Cerri ${ }^{11}$
}

\begin{abstract}
Resumo A sarcoidose representa uma doença inflamatória cuja etiologia permanece desconhecida. Acomete principalmente o interstício pulmonar, com formação de granulomas não caseosos e adenomegalia. Trata-se de enfermidade sistêmica, portanto, pode atingir qualquer órgão ou sistema, devendo ser lembrada no diagnóstico diferencial das doenças infecciosas, neoplásicas ou auto-imunes, isto porque, se diagnosticada corretamente, evita medicações ou cirurgias desnecessárias. Os autores relatam um caso de sarcoidose pulmonar com envolvimento hepático, no qual o paciente desenvolveu cirrose e trombose de veia porta. Unitermos: Fígado; Sarcoidose; Ultra-sonografia.
\end{abstract}

Abstract Liver involvement in sarcoidosis: a case report.

Sarcoidosis is an inflammatory disease whose etiology remains unknown. It is characterized by the formation of non-caseating granulomas and adenopathy, mainly in the pulmonary interstice. This is a systemic disease that may affect any organ or system and should be included in the differential diagnosis of infectious, neoplastic or autoimmune diseases as a correct diagnosis avoids unnecessary surgeries or administration of drugs. The authors report a case of pulmonary sarcoidosis with hepatic involvement in which the patient has developed cirrhosis and portal vein thrombosis.

Keywords: Liver; Sarcoidosis; Ultrasonography.

\section{INTRODUÇÃO}

A sarcoidose é uma doença inflamatória sistêmica de etiologia desconhecida e se

* Trabalho realizado no Centro de Diagnóstico do Hospital Sírio Libanês, São Paulo, SP, no Departamento de Radiologia da Faculdade de Medicina da Universidade Federal de Goiás e no Departamento de Doenças do Aparelho Digestivo do Hospital Araújo Jorge da Associação de Combate ao Câncer em Goiás, Goiânia, GO.

1. Médico Residente do Departamento de Radiologia da Faculdade de Medicina da Universidade Federal de Goiás.

2. Médicos Radiologistas do Hospital das Clínicas da Faculdade de Medicina da Universidade Federal de Goiás.

3. Professor Convidado do Departamento de Radiologia da Faculdade de Medicina da Universidade Federal de Goiás.

4. Professor Doutor do Departamento de Radiologia da Faculdade de Medicina da Universidade de São Paulo.

5. Chefe do Departamento de Doenças do Aparelho Digestivo do Hospital Araújo Jorge da Associação de Combate ao Câncer em Goiás.

6. Cirurgião do Departamento de Doenças Digestivas do Hospital Araújo Jorge da Associação de Combate ao Câncer em Goiás.

7. Acadêmico de Medicina da Faculdade de Ciências Médicas da Universidade do Vale do Sapucaí.

8. Acadêmicos de Medicina da Faculdade de Medicina da Universidade Federal de Goiás.

9. Acadêmico de Medicina da Faculdade de Medicina da Universidade de Ribeirão Preto.

10. Chefe do Departamento de Radiologia da Faculdade de Medicina da Universidade Federal de Goiás.

11. Professor Titular do Departamento de Radiologia da Faculdade de Medicina da Universidade de São Paulo.

Endereço para correspondência: Dr. Márcio Martins Machado. Rua Ruy Brasil Cavalcante, 496, Ed. Art-1, ap. 1001, Seto Oeste. Goiânia, Go, 74140-140. E-mail: marciommachado@ ibest.com.br

Recebido para publicação em 28/10/2004. Aceito, após revisão, em 15/3/2005. caracteriza pela formação de granulomas não caseosos que podem acometer qualquer órgão ou sistema. É mais comum da terceira à quinta décadas de vida, predomina na raça negra, e tem baixa prevalência na América do Sul quando comparada a outras regiões. O pulmão é mais comumente acometido, porém a ocorrência abdominal sem evidência de alterações pulmonares na radiografia de tórax pode ser encontrada em cerca de $25 \%$ a $38 \%{ }^{(\mathbf{1})}$.

Neste artigo, os autores relatam um caso de paciente portador de sarcoidose com manifestação pulmonar associada a alterações hepáticas e esplênicas.

\section{RELATO DO CASO}

Paciente de 47 anos de idade, recentemente diagnosticado como portador de sarcoidose pulmonar, desenvolveu hemorragia digestiva alta. Ao exame de endoscopia digestiva alta, foram identificados cordões varicosos de grosso calibre e com sinais de sangramento recente.

Ao exame de ultra-sonografia (US), foi identificado fígado com aspecto heterogêneo, bordas rombas e superfície irregular, com pequena redução volumétrica relativa do lobo hepático direito quando comparado com o esquerdo. Ao estudo Doppler, foi identificada trombose da veia porta (Figura 1). O baço estava aumentado, com ecotextura homogênea (Figura 2).

Foram feitas sorologias para hepatite B e $\mathrm{C}$, as quais foram negativas, e o paciente também não apresentava antecedente de etilismo. Foi realizada biópsia hepática, que revelou cirrose associada a granulomas não caseosos.

\section{DISCUSSÃO}

No abdome, a sarcoidose pode simular várias doenças infecciosas, neoplásicas ou auto-imunes, sendo assintomática ou com sintomas inespecíficos (febre, fadiga, perda ponderal e diarréia) $)^{(\mathbf{1})}$.

O fígado e baço são os órgãos abdominais mais acometidos (40\% a 60\%), com ou sem a formação de granulomas ${ }^{(\mathbf{1})}$. É mais característico o envolvimento das regiões periportais, em vez do comprometimento hepático propriamente dito ${ }^{(2)}$. Devido a este fato, podemos ter o desenvolvimento de hipertensão portal. Ocasionalmente, poderemos encontrar, também, trombose da veia porta com sinais de transformação 


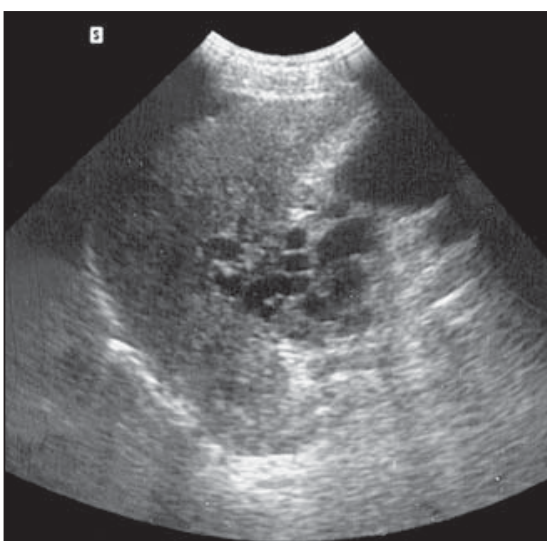

A

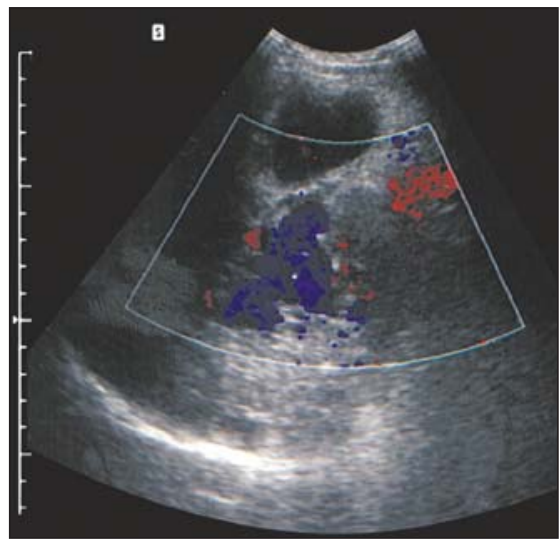

B

Figura 1. Trombose da veia porta em paciente com sarcoidose, observando-se os vasos colaterais periportais, caracterizando a transformação cavernomatosa. A: Exame em modo B. B: Exame com Doppler colorido.

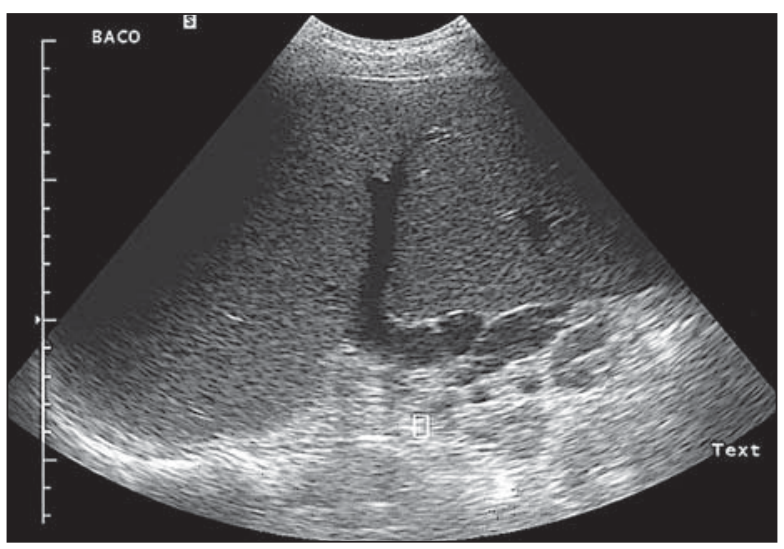

Figura 2. Sarcoidose. Esplenomegalia com vasos colaterais no hilo esplênico. cavernomatosa $^{(2)}$. Mesmo assim, a hipertensão portal não é usual ${ }^{(3)}$. Nas fases iniciais do envolvimento hepático na sarcoidose, os pacientes poderiam ser tratados com sucesso com corticóides ${ }^{(\mathbf{1})}$. Entretanto, se não houver tratamento efetivo, podese desenvolver fibrose periportal com o desenvolvimento de hipertensão portal irreversível ${ }^{(2,3)}$. Evolutivamente, o processo poderá avançar para o parênquima hepático e levar ao desenvolvimento de cirro$\mathrm{se}^{(\mathbf{1 , 3})}$. No presente caso, o paciente desenvolveu hipertensão portal provavelmente secundária à hepatopatia crônica (cirrose), conforme demonstrada pela biópsia hepática, tendo sido acometido também por trombose de veia porta, apresentando sinais de transformação cavernomatosa.

Radiologicamente, o achado mais comum é hepatomegalia (29\%) com fígado homogêneo ou heterogêneo. Nódulos focais podem ser observados em até $19 \%$ dos $\operatorname{casos}^{(\mathbf{1})}$. Esses, por sua vez, são acompanhados de hepatomegalia, adenopatia e nódulos esplênicos na maioria das vezes ${ }^{(\mathbf{1})}$. O uso de corticóide pode fazer os nódulos desaparecerem $^{(2)}$, porém a hepatomegalia permanece $^{(\mathbf{1})}$. No nosso paciente, não identificamos hepatomegalia, nódulos hepáticos ou esplênicos, ou linfonodomegalia ao exame ultra-sonográfico.

Embora o fígado possa ser normal ao ultra-som nos casos de sarcoidose com envolvimento hepático, alterações texturais difusas também têm sido descritas para estes casos. Como citado anteriormente, os granulomas usualmente são muito pequenos para serem identificados pelo exame ultra-sonográfico ${ }^{(2,3)}$. Ademais, ao ultrasom, poderíamos observar, ainda, aumento da ecogenicidade hepática ${ }^{(3)}$. Os nódulos, quando presentes, se mostram geralmente hipoecóicos em relação ao parênquima, embora casos hiperecóides tenham sido descritos $^{(\mathbf{1})}$. Na tomografia computadorizada (TC) após a administração do contraste endovenoso, os nódulos se apresentam como lesões hipoatenuantes e hipocaptantes. A ressonância magnética (RM) mostra lesões hipointensas em todas as sequiências pré-contraste e são mais conspícuas nas imagens precoces pós-gadolínio ponderadas em $\mathrm{T}^{(\mathbf{1})}$.

A esplenomegalia é encontrada de $25 \%$ a $60 \%$ dos pacientes com sarcoidose abdominal e está associada à presença de granulomas que normalmente são muito pequenos para serem visualizados na US, TC ou $\mathrm{RM}^{(\mathbf{1})}$. As calcificações seqüelas são incomuns, tanto no fígado quanto no baço, mas podem ser encontradas ${ }^{(\mathbf{3})}$. No paciente avaliados por nós, encontramos apenas esplenomegalia homogênea.

Apesar de a sarcoidose abdominal ser mais comum no fígado, baço e linfonodos, ela pode acometer qualquer órgão abdominal. Ademais, pode simular doenças neoplásicas, infecciosas ou auto-imunes em estudos radiológicos, devendo, portanto, ser incluída no diagnóstico diferencial das organomegalias abdominais ${ }^{(\mathbf{1})}$.

\section{CONCLUSÃO}

Os autores concluem que o envolvimento hepático na sarcoidose deve ser lembrado no acompanhamento de pacientes com sarcoidose pulmonar, podendo se manifestar com hipertensão portal e hemorragia digestiva alta, entendida no contexto possível de uma hepatopatia crônica (cirrose), que pode estar associada à sarcoidose.

\section{REFERÊNCIAS}

1. Warshauer DM, Lee JKT. Imaging manifestations of abdominal sarcoidosis. AJR 2004;182:15-28.

2. Machado MM, Rosa ACF, Cerri GG. Doenças hepáticas difusas, hipertensão portal e transplante de fígado. In: Cerri GG, Oliveira IRS, editores. Ultrasonografia abdominal. Rio de Janeiro, RJ: Revinter, 2002;55-124.

3. Mergo PJ, Ros PR. Imaging of diffuse liver disease. Radiol Clin North Am 1998;36:365-375. 